\title{
Primary Klebsiella pneumoniae Liver Abscess with Metastatic Spread to Lung and Eye, a North-European Case Report of an Emerging Syndrome
}

\author{
Sara K Sobirk ${ }^{1, *}$, Carsten Struve ${ }^{2}$ and Sanda G Jacobsson ${ }^{3}$ \\ ${ }^{I}$ Clinical Microbiology, University and Regional Laboratories Region Skåne s-221 85 Lund, Sweden \\ ${ }^{2}$ Department of Bacteriology, Mycology and Parasitology, Statens Serum Institut, 2300 Copenhagen S, Denmark \\ ${ }^{3}$ Department of Infectious Diseases, County Hospital of Helsingborg, s-251 87 Helsingborg, Sweden
}

\begin{abstract}
A syndrome of community acquired liver abscess caused by highly virulent hypermucoviscous, $r m p A$ positive Klebsiella pneumoniae strains occurs with high incidence in Asia. We here report a case complicated by metastatic infection, to our knowledge, the first one described in Northern Europe. This and other recently reported cases indicate the emergence of this severe syndrome outside of Asia.
\end{abstract}

Keywords: Klebsiella pneumoniae, metastatic infection, hypermucoviscous, rmpA.

\section{INTRODUCTION}

In recent years a high incidence of community-acquired primary liver abscess caused by highly virulent strains of Klebsiella pneumoniae has been documented in Taiwan and other Asian countries. [1-4]. A syndrome, with metastatic spread to eyes, meninges, brain and other sites, has been described, and is strongly associated with $K$. pneumoniae strains of capsular serotype K1 of a characteristic hypermucoviscous phenotype $[4,5]$. Two plasmid encoded virulence genes, rmpA (regulator of the mucoid phenotype) and the iron siderophore aerobactin, as well as two chromosomal encoded virulence genes, $k f u$ encoding an iron uptake system and allS associated with allatonin metabolism are significantly correlated with the high virulence of the invasive strains $[6,7]$. These highly virulent strains are so far rare outside of Asia, but case reports from USA, Canada, Spain and Belgium have been published lately [8-14].

We here report a Swedish patient with this emerging syndrome caused by a highly virulent $K$. pneumoniae isolate. This is, to our knowledge, the first case described in Northern Europe.

\section{CASE REPORT}

A 45-year old man of Vietnamese origin was admitted with a ten day history of fever, anorexia, fatigue and rightsided abdominal pain. He had no previous history of disease and no medication. At examination, he had signs of peritonitis, hypotension and a red and swollen left eye. He was initially treated with intravenous fluids and imipenem for suspected septicaemia. Pulmonary X-ray and CT-scan revealed right-sided pulmonary infiltrates with pleural effusion and a large mass in the liver with the appearance of multiple cysts.

*Address correspondence to this author at the Clinical Microbiology, University and Regional Laboratories Region Skåne s-221 85 Lund, Sweden; Tel: +46-46173287; E-mail: Sara.m.karlssonsobirk@skane.se
(Fig. 1) Examination of the eye showed marked chemosis, absence of light reflex and intravitreal hemorrhage. Empiric treatment for endophtalmitis was given intravitreously (ceftazidime and vancomycin). Cultures of blood, urine, aspirates from liver and pleural effusion all grew K. pneumoniae sensitive to imipenem and ceftazidime. Cultures that were taken from the eye, after initiation of intravenous therapy, were negative. Despite adequate treatment and continuous drainage of both liver abscess and empyema, repeated cultures from these sites showed the same mucoid strain of $K$. pneumoniae up to 23 days after initiation of intravenous antibiotics. Two repeated blood cultures taken one week after initiation of therapy were negative. Following three weeks of intravenous therapy, the patient was treated with oral ciprofloxacin for another five weeks. His recovery, although slow, corresponded well with repeated CT-scans of lungs and liver showing regress of the infection. Although the pain in his left eye decreased, his vision is permanently damaged, and he is now blind on the affected eye. The patient had immigrated to Sweden 8 years previously, passing a refugee-camp in Hong Kong, and had been back to visit Vietnam once, 10 months before start of symptoms. Initial investigations of the patient therefore included TB-analyses and a broad parasitic screen, all found to be negative.

The K. pneumoniae isolate that caused the infection was found to express the hypermucoviscous phenotype as shown by the formation of mucoviscous strings when a loop was passed through a colony (Fig. 2). Capsular serotyping at the International Eschericia coli and Klebsiella Reference Centre (WHO), Statens Serum Institut revealed that the isolate belongs to the $\mathrm{K} 1$ serotype which was confirmed by detection of the K1 serotype specific gene $\operatorname{mag} A$ as previously described (Fig. 3) [15]. Furthermore the isolate was positive for all four virulence genes $r m p A$, aerobactin, $k f u$ and allS as revealed by polymerase chain reaction using specific primers (Fig. 3) [5]. Thus the isolate exhibited similar characteristics 


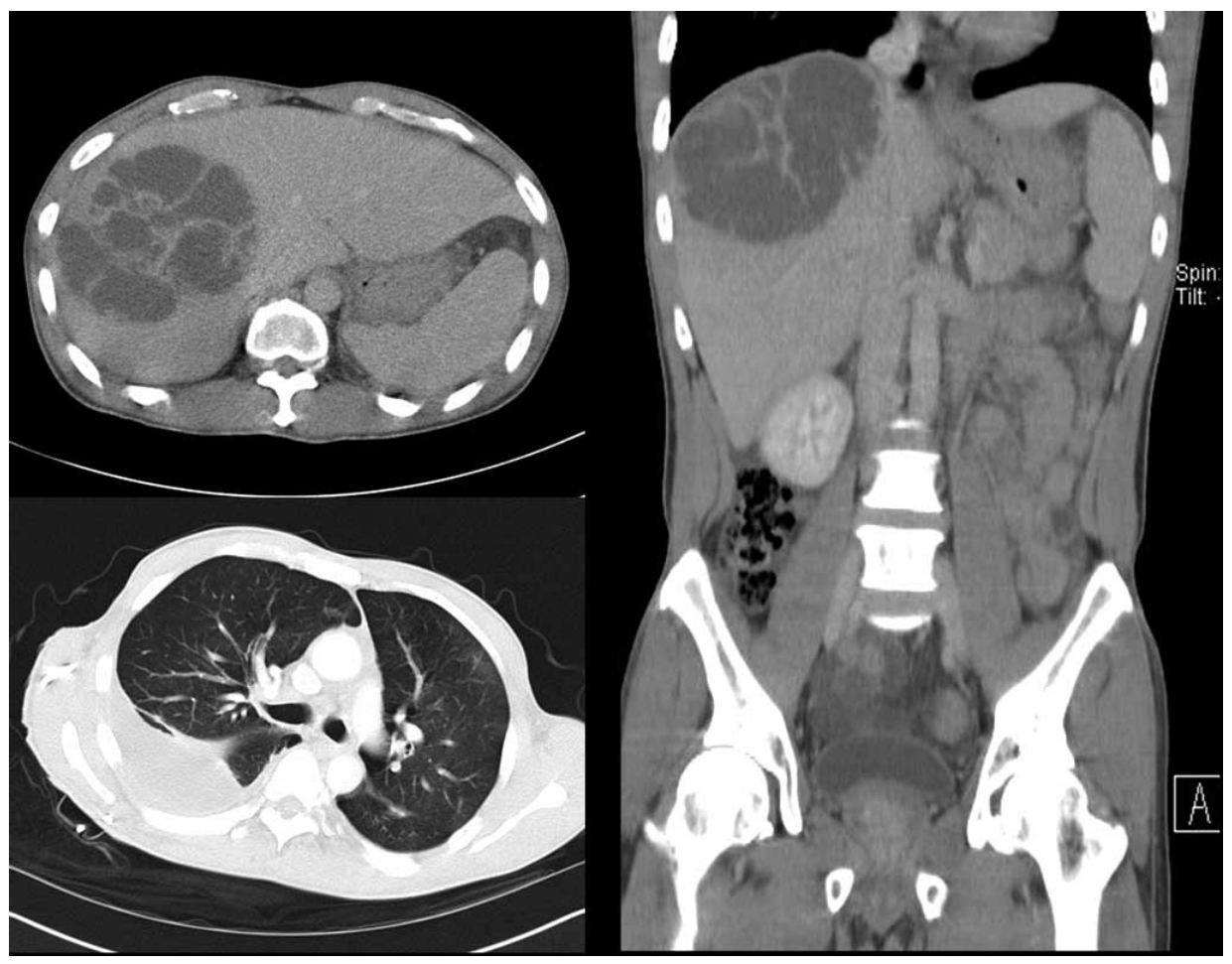

Fig. (1). CT-scans showing liver abscess and right sided empyema.

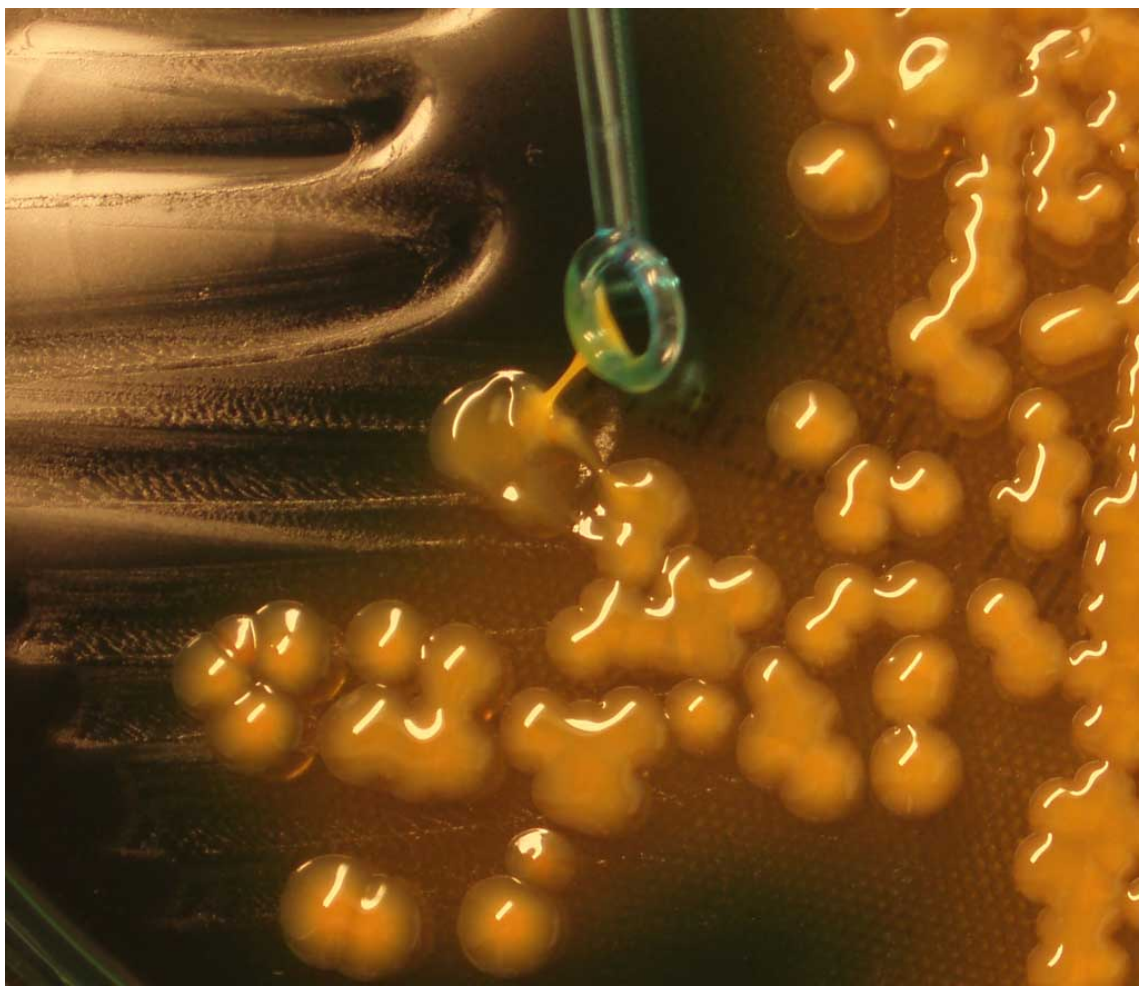

Fig. (2). Plate culture demonstrating the hypermucoviscous phenotype of the isolate.

as the highly virulent $K$. pneumoniae isolates that have so far mainly been observed in Asia.

\section{DISCUSSION}

The rising incidence of $K$. pneumoniae community acquired primary liver abscess in otherwise healthy indi- viduals caused by highly virulent strains and often complicated by severe metastatic infections is disturbing [2, 3, 14]. Whereas this emerging infectious disease so far has been observed mainly in Asia, a rising number of cases from other geographic regions indicate that these infections may develop into a world wide problem. It is remarkable that the 


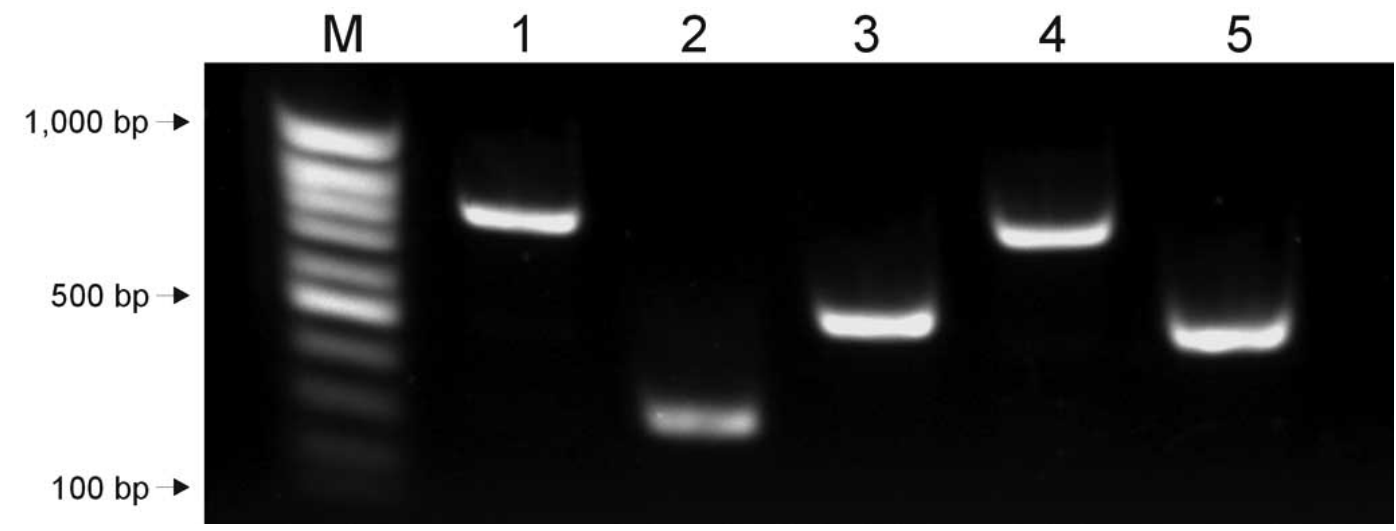

Fig. (3). Detection of $\operatorname{mag} A$ (lane 1), rmpA (lane 2), aerobactin (lane 3), $k f u$ (lane 4) and allS (lane 5). PCR amplifications were performed by use of specific primers $(5,15)$.

majority of cases outside Asia have involved patients of Asian ethnicity. The reasons for the predominance of this syndrome in patients of Asian ethnicity are not known, but suggest that genetic susceptibility may play an important role. Alternatively, it could be speculated that there may be a food source of the virulent bacteria. Cases with no history of traveling to Asia have been reported demonstrating that highly virulent $K$. pneumoniae strains are present outside of Asia $[10,12,13]$. However, although our patient had only had Swedish and Vietnamese contacts, he may have been colonized with the strain in Vietnam 10 months before onset of symptoms.

Patients with a recent history of travelling in foreign countries are often thoroughly investigated for infections with virus, bacteria or parasites that are typical for the region visited. Bacterial species that are common all over the world can, with the same antibiotic susceptibility-pattern, vary geographically in regard to virulence-factors corresponding to clinical picture and outcome. A connection between the causative agent, the region visited and the clinical picture is easily overlooked if analyses of these bacterial strains are restricted to identification to species-level and susceptibilitytesting. With this case report, we would like to highlight an emerging syndrome caused by a bacterial species common in Europe, but of a serotype and with specific virulence genes still rare outside of Asia.

\section{ACKNOWLEDGEMENTS}

We thank Dr Johan Rydberg at the Department of Clinical Microbiology and Immunology at the University Hospital in Lund, for his support and valuable comments.

\section{REFERENCES}

[1] Wang JH, Liu YC, Lee SS, et al. Primary liver abscess due to Klebsiella pneumoniae in Taiwan. Clin Infect Dis 1998; 26: 14348 .

[2] Tsai FC, Huang YT, Chang LY, Wang JT. Pyogenic liver abscess as endemic disease, Taiwan. Emerg Infect Dis 2008; 14: 1592-600.
[3] Chung DR, Lee SS, Lee HR, et al. Emerging invasive liver abscess caused by $\mathrm{K} 1$ serotype Klebsiella pneumoniae in Korea. J Infect 2007; 54: 578-83.

[4] Fung CP, Chang FY, Lee SC, et al. A global emerging disease of Klebsiella pneumoniae liver abscess: is serotype $\mathrm{K} 1$ an important factor for complicated endophthalmitis? Gut 2002; 50: 420-4.

[5] Fang CT, Lai SY, Yi WC, Hsueh PR, Liu KL, Chang SC. Klebsiella pneumoniae genotype K1: an emerging pathogen that causes septic ocular or central nervous system complications from pyogenic liver abscess. Clin Infect Dis 2007; 45: 284-93.

[6] $\mathrm{Yu}$ VL, Hansen DS, Ko WC, et al. Virulence characteristics of Klebsiella and clinical manifestations of $K$. pneumoniae bloodstream infections. Emerg Infect Dis 2007; 13: 986-93.

[7] Yu WL, Ko WC, Cheng KC, Lee CC, Lai CC, Chuang YC. Comparison of prevalence of virulence factors for Klebsiella pneumoniae liver abscesses between isolates with capsular $\mathrm{K} 1 / \mathrm{K} 2$ and non-K1/K2 serotypes. Diagn Microbiol Infect Dis 2008; 62: 16.

[8] Nadasy KA, Domiati-Saad R, Tribble MA. Invasive Klebsiella pneumoniae syndrome in North America. Clin Infect Dis 2007; 45: e25-e8.

[9] Fang FC, Sandler N, Libby SJ. Liver abscess caused by $m a g A+$ Klebsiella pneumoniae in North America. J Clin Microbiol 2005; 43: 991-2.

[10] Frazee BW, Hansen S, Lambert L. Invasive Infection With Hypermucoviscous Klebsiella pneumoniae: multiple cases presenting to a single emergency department in the United States. Ann Emerg Med 2009; 53: 639-42

[11] Keynan Y, Karlowsky JA, Walus T, Rubinstein E. Pyogenic liver abscess caused by hypermucoviscous Klebsiella pneumoniae. Scand J Infect Dis 2007; 39: 828-30.

[12] Karama EM, Willermain F, Janssens X, et al. Endogenous endophthalmitis complicating Klebsiella pneumoniae liver abscess in Europe: case report. Int Ophthalmol 2008; 28: 111-3.

[13] Gomez C, Broseta A, Otero JR, Chaves F. Primary pyogenic liver abscess caused by magA + Klebsiella pneumoniae in Spain. Clin Microbiol News1 2007; 29: 100-2.

[14] Lederman ER, Crum NF. Pyogenic liver abscess with a focus on Klebsiella pneumoniae as a primary pathogen: an emerging disease with unique clinical characteristics. Am J Gastroenterol 2005; 100: 322-31.

[15] Struve C, Bojer M, Nielsen EM, Hansen DS, Krogfelt KA Investigation of the putative virulence gene $\operatorname{mag} A$ in a worldwide collection of 495 Klebsiella isolates: magA is restricted to the gene cluster of Klebsiella pneumoniae capsule serotype K1. J Med Microbiol 2005; 54: 1111-3. 